\title{
Recurrence of Cancer After Endoscopic Ablation of Barrett's Esophagus: Is the Elephant in the Room...Persistent Ongoing Reflux?
}

\author{
Seth A. Gross • Prateek Sharma
}

Published online: 23 March 2014

(c) Springer Science+Business Media New York 2014

Intestinal metaplasia of the esophageal epithelium, termed Barrett's esophagus (BE), usually precedes the development of esophageal adenocarcinoma. Due to the high morbidity associated with surgical resection, endoscopic ablative therapy for BE patients with premalignant highgrade dysplastic epithelial features and intra-mucosal cancers (IMC) is increasingly popular [1]. Overall, although endoscopic therapy is usually initially effective, patients can still develop recurrent BE or cancer after endoscopic ablation. Recurrence of $\mathrm{BE}$ after radiofrequency ablation therapy (RFA) has been reported in $13-35 \%$ of the patients, with the majority of recurrences manifest as intestinal metaplasia without dysplasia [2-4]. The factors contributing to recurrence include ineffective ablative therapy, uncontrolled gastro-esophageal reflux disease (GERD), long length of the metaplastic epithelium, and advanced age. The clinical challenge is to identify predictors for patients who relapse after ablation therapy with recurrent $\mathrm{BE}$ or with cancer.

In this issue of Digestive Diseases and Sciences, Yasuda et al. [5] assess the clinical predictors associated with de novo adenocarcinoma development after successful endoscopic ablation therapy. In a retrospective analysis of their 15-year experience of treating BE-HGD and IMC with ablation modalities including photodynamic therapy, RFA, cryotherapy, fulguration, and endoscopic mucosal resection

\footnotetext{
S. A. Gross $(\bowtie)$

Division of Gastroenterology, NYU Langone Medical Center, NYU School of Medicine, New York, NY, USA

e-mail: Seth.Gross@nyumc.org

P. Sharma

Division of Gastroenterology and Hepatology, Veterans Affairs Medical Center, University of Kansas School of Medicine, Kansas City, KS, USA
}

(EMR) with the majority of the patients treated with more than one type of therapy. Of a cohort of 223 patients who had undergone ablative treatment for BE with dysplasia or IMC, 183 patients met final study analysis criteria. After endoscopic therapy, patients were placed into a rigorous surveillance program with endoscopy repeated initially every 2-3 months, then every 6 months, and then annually. After successful initial ablation, adenocarcinoma developed in 20 patients $(11 \%)$, the majority with IMC and the median time of recurrence after initial therapy was 11.5 months (range 3-109 months). There were no statistical differences in rate of recurrence among all types of ablation therapies (PDT vs RFA), or who received a single modality compared with patients who were treated with multi-modality therapy. A significant endoscopic finding during surveillance of this cohort was the presence and size of a hiatal hernia, which was present in 76 and $51 \%$ of patients with and without recurrent cancer, respectively. Furthermore, the group with recurrent cancer had a hiatal hernia $>4 \mathrm{~cm}$ in $38 \%$ compared to $12 \%$ in the group without recurrence $(p=0.0007)$ Finally, recurrence was also associated with HGD or adenocarcinoma after the initial ablation session.

Though this report provides some insight into predictors of recurrence, it has several limitations. First, the study took place during the evolution of endoscopic ablative therapies with resultant heterogeneity of therapeutic modalities studied. Second, current clinical practice often involves EMR in combination with RFA. Third, data from retrospective single-center design experience may be subject to biases and statistical weaknesses less present in large prospective multi-center studies. Fourth, esophageal $\mathrm{pH}$ testing was not used to document whether increased esophageal acid exposure accompanied the presence of a hiatal hernia. Finally and most importantly, the small 
sample size of recurrent cancers is not sufficient to enable robust multi-variant analysis of the predictive risk factors associated with recurrences.

Despite these limitations, Yasuda et al. identified the presence and size of a hiatal hernia as a significant factor associated with the development of subsequent cancer after the completion of endoscopic therapy. The contributions of ongoing esophageal reflux and hiatal hernia to the pathogenesis of BE, BE dysplasia, and cancer, and the success of ablation therapy have been reported in several publications. Hiatal hernia, in a recent meta-analysis, was identified as a risk factor for the development of BE [6]. Large hiatal hernias are associated with longer BE segment length and impaired healing of the esophagus after ablation secondary to continued acid exposure [7]. The presence of a hiatal hernia is linked to incomplete eradication of $\mathrm{BE}$ after ablation therapy, since any factor that promotes continued acid reflux into the distal esophagus after ablation therapy is associated with persistent $\mathrm{BE}$ [8].

In summary, the study by Yasuda et al. contributes to the existing literature suggesting that persistent, ongoing gastro-esophageal reflux due to the presence of large hiatal hernias is a predictor of subsequent recurrence of cancer after successful endoscopic ablation therapy. As in the highlighted paper, recurrence rates of 3-20\% after successful ablation of subjects with either BE or IMC have been reported in several studies $[9,10]$. The study raises several questions such as the need for pre-ablation 24-h pH and impedance studies to document acid control in the presence of a hiatal hernia, and the efficacy of pre-ablation high dose of proton pump inhibitor therapy or surgical correction of the hernia to normalize esophageal acid exposure.

Furthermore, since persistent HGD or IMC after initial ablation was associated with future recurrence, an incomplete response to initial therapy may be augmented by persistent acid reflux due to the hiatal hernia. In considering the Yasuda study and others, it may be time to take a step back and consider, before offering ablative therapy, whether acid reflux and its promoters, especially a large hiatal hernia, should be addressed and corrected prior to ablation as a means of ensuring the ultimate success of endoscopic ablative therapy of BE.

\section{References}

1. Spechler SJ, Sharma P, Souza RF, Inadomi JM, Shaheen NJ. American Gastroenterological Association medical position statement on the management of Barrett's esophagus. Gastroenterology. 2011;140:1084-1091.

2. Orman ES, Li N, Shaheen NJ. Efficacy and durability of radiofrequency ablation for Barrett's Esophagus: systematic review and meta-analysis. Clin Gastroenterol Hepatol. 2013;11:1245-1255.

3. Gupta M, Iyer PG, Lutzke L, et al. Recurrence of esophageal intestinal metaplasia after endoscopic mucosal resection and radiofrequency ablation of Barrett's esophagus: results from a US Multicenter Consortium. Gastroenterology. 2013;145:79-86.

4. Guarner-Argente C, Buoncristiano T, Furth EE. Long-term outcomes of patients with Barrett's esophagus and high-grade dysplasia or early cancer treated with endoluminal therapies with intention to complete eradication. Gastrointest Endosc. 2013;77:190-199.

5. Yasuda K, Choi SE, Nishioka NS. Incidence and predictors of adenocarcinoma following endoscopic ablation of Barrett's esophagus. Dig Dis Sci. (Epub ahead of print). doi:10.1007/ s10620-013-3002-5.

6. Andrici J, Tio M, Cox MR, Eslick GD. Hiatal hernia and the risk of Barrett's esophagus. J Gastroenterol Hepatol. 2013;28:415-431.

7. Korst RJ, Santana-Joseph S, Rutledge JR. Effect of hiatal hernia size and columnar segment length on the success of radiofrequency ablation for Barrett's esophagus: a single-center, phase II clinical trial. J Thorac Cardiovasc Surg. 2011;142:1168-1173.

8. Krishnan K, Pandolfino JE, Kahrilas PJ, et al. Increased risk for persistent intestinal metaplasia in patients with Barrett's esophagus and uncontrolled reflux exposure before radiofrequency ablation. Gastroenterology. 2012;143:576-581.

9. Shaheen NJ, Sharma P, Overholt BF, et al. Radiofrequency ablation in Barrett's esophagus with dysplasia. $N$ Engl J Med. 2009:360:2277-2288.

10. Haidry RJ, Dunn JM, Butt MA, et al. Radiofrequency ablation and endoscopic mucosal resection for dysplastic Barrett's esophagus and early esophageal adenocarcinoma: outcomes of the UK National Halo RFA Registry. Gastroenterology. 2013;145:87-95. 